\title{
IgG4-related sclerosing mesenteritis and its possible relation to papillary thyroid carcinoma in a 27-year-old Egyptian woman.
}

\author{
Hany El-Saadany* \\ Air Force Specialized Hospital, New Cairo, Egypt
}

\begin{abstract}
Sclerosing mesenteritis (SM) is a rare fibro inflammatory disease unknown etiology involves the small bowel mesentry, most frequently observed in middle aged and older men. Recently, it was reported to be closely related to IgG4-RD. Many articles reported a close relation between IgG4-related and thyroid diseases including Hadhimoto's thyroiditis and papillary thyroid cancer. This report describes a lady with sclerosing mesenteritis (SM) proved serologically as well as histo-pathologically to be associated with IgG4-RD, with a past history of Hashimoto's thyroiditis then a hemithyroidectomy was done to the patient for multi nodular goiter whose pathology revealed papillary adenocarcinoma and treated accordingly. These findings support the previous reports relating IgG-RD with sclerosing mesenteritis, Hashimoto's thyroiditis and papillary thyroid carcinoma.
\end{abstract}

Keywords: IgG4-RD, Sclerosing Mesentritis (SM), Papillary thyroid carcinoma, Intestinal perforation, Intestinal obstruction.

\section{Introduction}

Sclerosing mesenteritis (SM) is a non-specific inflammatory mass forming lesion in the mesenteric connective tissue that is characterized by variable degrees of fibrosis, chronic inflammation and fat necrosis mainly affecting the mesentry of the small bowel [1-4]. Possible risk factors include malignancy, autoimmune diseases, ischemia and history of previous surgery [5-10] due to its similarities clinically and radio logically with malignancy, sometimes they are indistinguishable [3,6,7]. Most recently, Sclerosing mesenteritis has been recognized to be associated with IgG4-RDand the term IgG4 related mesenteritis has since been incorporated into this spectrum of diseases $[5,6]$.

We report a very rare case of IgG4-related sclerosing mesenteritis with multiple bowel perforations that underwent multiple surgical procedures starting from resection and anastomosis to excision of a mass in the jeugenal mesentry to another exploration in addition to the hemi thyroidectomy done before for a multi nodular goiter proved pathologically to be a papillary thyroid adenocarcinoma.

\section{Case Report}

A-27 year-old woman,who had a resection and anastomosis operation 2 months ago at the police hospital in Cairo for multiple intestinal perforations with peritonitis presented at the emergency room of the Air force specialized hospital in Cairo by an acute abdominal pain, severe recurrent vomiting and pain allover. An erect and supine plain $\mathrm{x}$ ray abdomen were done which were normal, there was an electrolyte imbalance the patient was admitted at the surgical department where a CT scanning of the abdomen revealed an irregular shaped mass $4 \mathrm{~mm}$ in diameter over the jeugenal mesentryan initial nasogastric tube placed for decompression she was made nothing by mouth (NPO) and started total parenteral nutrition (TPN), systemic antibiotics and pain killers. Elevated temperature, white blood cells (17.000), ESR (55), CRP (194) moderate microcytic hypochromic anemia (Hb: 9 gm), hypo albuminemia $(2.6 \mathrm{mg} / \mathrm{dL})$ Vitamin D level was $50 \%$ of the reference range and pain allover are the main picture at that time. She is treated supportively till the surgeon explored her due to the persistence of recurrent severe abdominal pain and vomiting, the jeugenal mesenteric mass was existed and sent for histopathology. Still after excision of the mass, the abdominal pain and vomiting recurred vigorously for which arheumatological consultation for us was done. Upon taking history and examination of the patient I noticed her previous investigate on refer to a Hashimoto's thyroiditis and an old scar of thyroid operation the patient mentioned that it was for a multi nodular goiter proved pathologically to be a papillary adenocarcinoma. Our new investigations revealed elevated circulating plasma blasts gated to $\mathrm{CD} 38$, CD138, CD27, CD19 and CD20 in a flow cytometry of peripheral blood with a picture consistent with IgG4-RD also, there were elevated IgG4 titer and $\mathrm{IgG} 4 / \mathrm{IgG}$ titer ratio $[11,12]$. Without waiting the histo pathological results, we recommended a 3 days pulse of methylprednisolone IV infusion and cyclophosphamide by IV infusion to be recurred every month for 6 months according to their commended doses also a replacement for vitamin D by 200.000 IU every 3 weeks. Two days later, the patient stopped vomiting with marked amelioration of the abdominal pain, she started a maintenance of $40 \mathrm{mg}$ prednisone/d and discharged 2 weeks later after she was able to eat and walk supported waiting for another operation for dissection of intestinal adhesions and an abdominal wall fistula later on. She is now doing well. The biopsy of the mesenteric mass revealed fibroblasts among fat cells and store form proliferation of spindle cells with many inflammatory cells. 


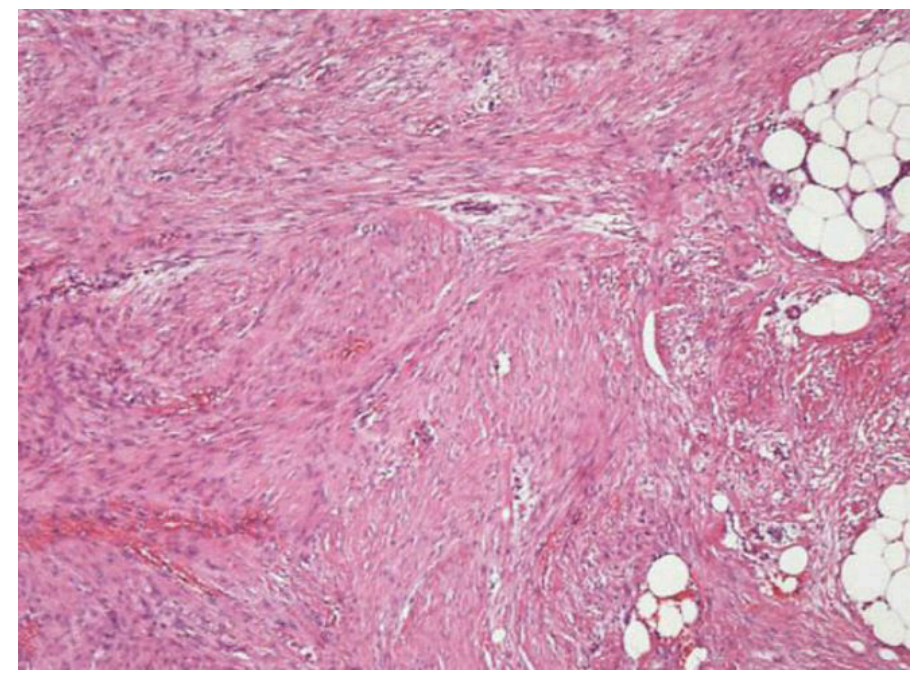

Figure 1. Histopathology of the mesenteric mass.

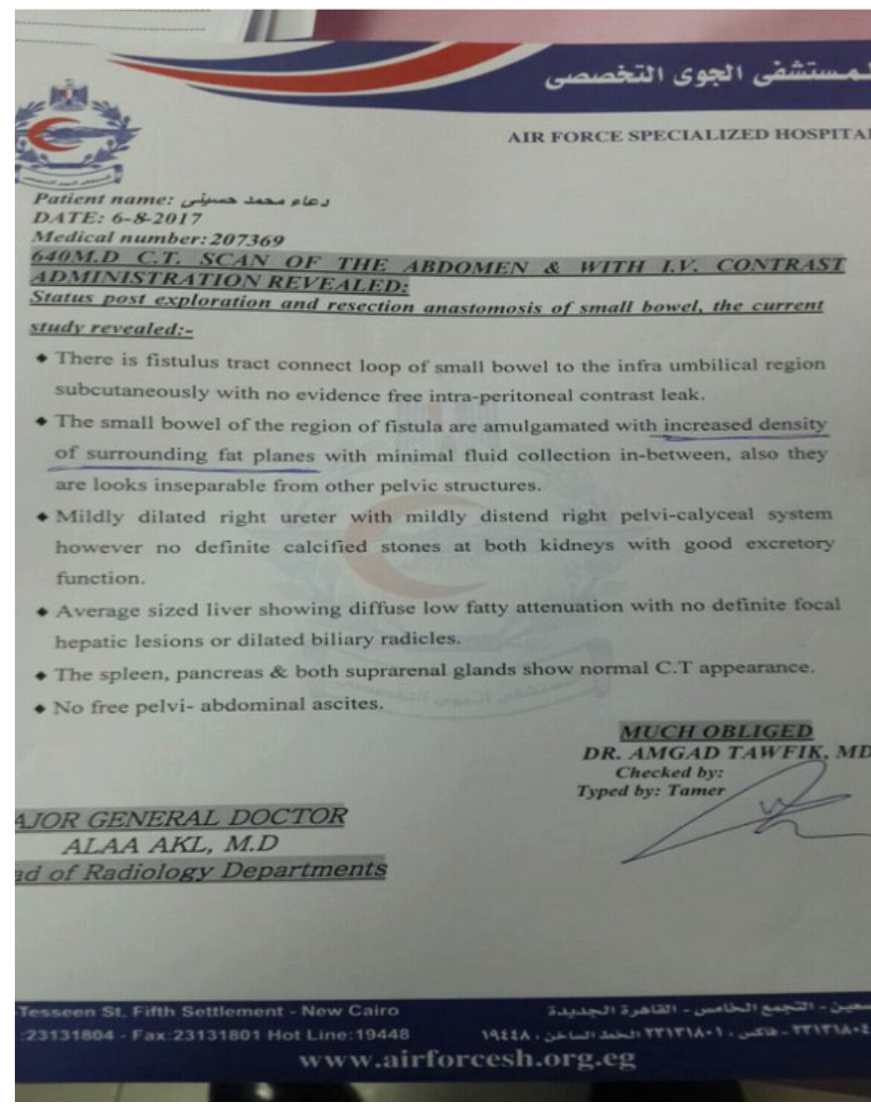

Figure 2. Follow up CT scanning report of the abdomen.

\section{Discussion}

This report describes a woman with an IgG4 related Sclerosing mesenteritis. Among the implicated etiologies are previous surgery (40\% to $70 \%)$, TB, Autoimmune diseases and vascular insufficiency. SM may be an IgG4 -RD $[5,8]$ that dramatically responds to corticosteroid treatment. IgG4-RD is characterized by organ involvement and nodular/hyperplastic lesions in various organs due to marked infiltrations of lymphocytes and IgG4 positive plasma cells, as well as to fibrosis. Diagnosis of IgG4 -RD comprises in addition to the clinical; imaging, serum and histopathological examination: (1) Clinical examination showed show characteristic diffuse/local swellings or masses in single or multiple organs. (2) Hematological involved serum IgG4 concentration $(>135 \mathrm{mg} / \mathrm{dL})$, elevated circulating plasma blasts gated toCD38, CD27, CD27, CD20, CD19 [9-12]. Histopathology examination showed show marked lymphoplasmacytic infiltrations, obstructive phlebitis, dense fibrosis and an infiltration of IgG4 positive plasma cells. Searching the internet for the relation between IgG4-RD and Sclerosing mesenteritis revealed eight cases ofIgG4 related Sclerosing mesenteritis $[5,8,11]$ most of them are symptomatic with abdominal pain or a palpable abdominal mass. Abdominal CT scan is very important for an accurate diagnosis. Without histopathology, the following findings are important for 
suggesting SM: (1) Hyper attenuating mesenteric fat. (2) Well defined soft tissue nodules less than $5 \mathrm{~mm}$ in diameter surrounded by a fatty halo (Fat ring sign). (3) Temporal pseudo capsule. The previous reports of association between IgG4-RD and Hashimoto's thyroiditis and Papillary thyroid carcinoma as well as this lady need further large studies to clarify this association (Figures 1 and 2) [10].

\section{Management}

Ranges from left untreated in asymptomatic patients to surgical exploration in life threatening complications as bowel obstruction or perforation or if there is a suspicion of malignancy. Medical treatment including corticosteroids, tamoxifen, cyclophosphamide and azathioprine has also shown good results especially corticosteroids if SM is due to IgG4$\mathrm{RD}$ [1-3]. Building on the specific treatment recommendations given to patients by their doctor, meetings between the care manager and the patient could take a broad perspective and consider the medical, social, behavioral, and emotional impact that living with a chronic conditioner reducing a health risk might have on the patient's quality of life. Each of the care manager's interventions was intended to increase patient empowerment, supporting patients to develop the confidence to effectively self-manage their health. Throughout the course of treatment, the care manager would provide support to the patient in implementing actions based on the GP recommendations or in taking steps to make the lifestyle changes needed to improve health or lower health risk. Unfortunately, in this case like many others we don't have this system of care manager, social workers, actually, her husband which is a police officer did well the role of care manager [13]. We recommend inclusion of IgG related sclerosis mesenteritis in every case of IgG4 recurrent abdominal pain and/or mesenteric masses.

\section{Conclusion}

IgG4-RD may be associated with SM, Hashimoto's thyroiditis, and papillary thyroid carcinoma. Although SM may be difficult to be diagnosed without histopathology, yet, it should be considered in any abdominal pain or mesentric mass to avoid unnecessary surgical interventions especially with good response to corticosteroids. Also taking any thyroid abnormality in patients with IgG4-RD seriously for fear of the possibility of papillary thyroid carcinoma.

\section{Competing Interests}

The author declares that there are no competing interests.

\section{Consent for Publication}

A written consent was taken from the patient.

\section{References}

1. Emory TS, Monihan JM, Carr NJ, et al. Sclerosing mesenteritis, mesenteric panniculitis and mesenteric lipodystrophy a single entity? Am J Surg Pathol.1997;21:392-398.

2. Akram S, Pardi DS, Schaffner JA, et al. Sclerosing mesenteritis: Clinical features, treatment and outcome in a ninety two patients. Clin Gastroenterol Hepatol. 2007;5:589-596.

3. Vlashos K, Archontovasilis F, Falidas E, et al. Sclerosing mesenteritis: Diverse clinical presentations and dissimilar treatment options. A case series and review of literature. Int Arch Med. 2011;4:17.

4. Toshifumi W, Terai S, Tsukada T, et al. Sclerosis mesenteritis mimicking metachronous peritoneal metastases from descending colon adenocarcinoma. World J Surg Oncol. 2017; 15:142 .

5. Chen TS. Are tumefactive lésions classified as sclerosis mesenteritis a subset of IgG4 related sclerosing disorder? J Clin Pathol. 2008;61:1093-1097.

6. Nomuro Y, Naito Y, Eriguchi N, et al. A case of IgG4 related sclerosing mesenteritis. Pathol Res Pract. 2011;207:518-521.

7. Salvarani C, Valli R, Boiardi L, et al. IgG4 associated sclerosing mesenteritis. Clin Exp Rheumatol. 2011;29:579-580.

8. Minato H. IgG4 related related sclerosing mesenteritis: A rare mesenteric disease of unknown etiology. Pathol Int. 2012;62:281-286.

9. Kamisawa T, Okamoto A. IgG4 related sclerosing disease. World J Gastroenterology. 2008;14:3948-3955.

10. Yang Y, Zhang J, Lu G, et al. Clinical relationship between IgG4 related disease, Hashimoto's thyroiditis and papillary throid carcinoma. J Clin Endocrinol. 2017;1516-1523.

11. Hasosah M, Satti MB, Yousef YA, et al. IgG4 related sclerosing mesenteritis in a seven year old Saudi girl. Saudi J Gasroentetol. 2014;20:385-388.

12. El-Saadany HA. Case series: Association between elevated circulating plasmablasts and their gated flow-cytometric picture in a cohort of IgG4-RDcases. J Clin Cell Immunol. 2017.

13. Ciccone MM, Aquilino A, Cortese F, et al. Feasibility and effectiveness of a disease and care management model in the primary health care system for patients with heart failure and diabetes (Project Leonardo). Vasc Health Risk Manag. 2010;6:297-305.

\section{*Correspondence to:}

Hany El-Saadany

Air Force Specialized Hospital

New Cairo,

Egypt

Tel: 02-23131804,+20 19448

E-mail: hanyelsadany@yahoo.com 